\title{
Seasonal affective disorder
}

\section{Rodin \& C. Thompson}

Seasonal affective disorder (SAD) is a recurrent depressive disorder in which episodes occur at a particular time of year. The most frequent variant is winter SAD in which patients become depressed during the autumn or early winter, with remission or conversion to hypomania the following spring. Recognition of winter SAD is important because the diagnosis predicts a good response to the novel treatment of phototherapy. One other variant, summer SAD, has also been described and we will discuss this condition separately. Otherwise, we will confine our discussion to the winter variant which we will refer to as SAD.

\section{Diagnosis and classification}

\section{Seasonal affective disorder}

ICD-10 clinical guidelines do not include specific criteria for seasonal affective disorder. However, specific criteria are included in DSM-IV and the research version of ICD-10 (see Boxes 1 and 2). Both systems require there to have been recurrent episodes of mood disorder which meet standard diagnostic criteria. These episodes must show a regular temporal pattern and must substantially outnumber non-seasonal episodes. What constitutes a regular temporal pattern is not defined in DSM-IV, whereas in ICD-10, onset and remission must occur within separate 90-day periods: an example of this would be a recurrent depressive disorder with onset during September-November and remission during March-May. The other important difference between the two systems is that ICD-10 stipulates that seasonal episodes must have occurred for three or more consecutive years, whereas DSM-IV requires that seasonal episodes must have occurred in each of the past two years, in the absence of non-seasonal episodes.

Both these sets of diagnostic criteria represent a change from DSM-III-R which specified that onset should be during a particular 60-day period each year with remission or conversion to hypomania occurring during a subsequent 60 -day period. These criteria were loosened because they have been shown to exclude cases with a favourable response to phototherapy (Dittman et al, 1994). However, many phototherapy trials have used DSM-III-R criteria to define cases and so there is a narrower evidence base for use of phototherapy in SAD as defined by DSM-IV and ICD-10.

\section{Sub-syndromal SAD}

Sub-syndromal SAD is a term coined to describe a group of patients who present with the usual

Box 1. ICD-10 provisional criteria for seasonal affective disorder

Could be applied to mood (affective) disorder, categories F30-33

Three or more episodes of mood (affective disorder) must occur with onset within the same 90-day period of the year for three or more consecutive years

Remissions also occur within a particular 90-day period of the year

Seasonal episodes substantially outnumber any non-seasonal episodes that may occur

Chris Thompson is Professor of Psychiatry at the University of Southampton. He has been at the forefront of SAD research for the past 10 years. Ian Rodin is a Clinical Lecturer in psychiatry (Department of Psychiatry, University of Southampton, Royal South Hants Hospital, Brintons Terrace, Southampton SO14 OYG) and has been a co-worker on much of this research. They have both developed expertise in the assessment and management of SAD through their work within the Southampton Mood Disorders Service, which receives secondary and tertiary referrals from throughout the UK. 
Box 2. DSM-IV criteria for seasonal pattern specifier

Seasonal pattern can be applied to the pattern of major depressive episodes in bipolar I disorder, bipolar II disorder, or major depressive disorder, recurrent

There has been a regular temporal relationship between the onset of major depressive episodes and a particular time of the year (e.g. regular appearance of the major depressive episode in autumn or winter)

Do not include cases in which there is an obvious effect of season-related psychosocial stressors (e.g. regularly being unemployed each winter)

Full remissions or a change from depression to mania or hypomania also occur at a characteristic time of year (e.g. depression disappears in the spring)

In the past two years, two major depressive episodes have occurred that demonstrate the temporal seasonal relationships defined in the above two criteria and no non-seasonal major depressive episodes have occurred during that same period

Seasonal major depressive episodes substantially outnumber the non-seasonal major depressive episodes that may have occurred over the individual's lifetime

clinical picture of SAD but whose seasonal depressive symptoms fall short of criteria for major depressive disorder. This syndrome is alluded to in the text of DSM-IV and there is evidence from one study that it predicts a favourable response to phototherapy (Kasper et al, 1989a). Otherwise, the syndrome has not been well characterised.

\section{Validity of the diagnosis}

Is there really a distinct seasonal subtype of recurrent mood disorder? Seasonal and nonseasonal depression certainly differ in a number of ways and a comparison of several factors is shown in Table 1 . However, these findings may reflect the wide publicity the syndrome has received, leading to systematic bias in reporting and recording of symptoms and their course. Against this, there have been a number of reports of patients in whom retrospective examination of contemporaneous case notes revealed a seasonal pattern of recurrent depression over many years. The validity of the syndrome is also supported by epidemiological studies which showed that the prevalence of major depressive disorder in the general population and in primary care varies with season, occurring most often during winter (Thompson, 1989).

Studies of SAD also give some support for the biological basis of the syndrome. Abnormalities in the timing of melatonin secretion (Lewy et al, 1987) have been found in SAD, and suppression of melatonin by light has been shown to be increased during winter depressive episodes and then decreased during remission the following summer (Thompson et al, 1990). However, such findings often fail to be replicated in subsequent studies, possibly because of variations in methodology. Another biological abnormality identified in SAD is the cortisol and prolactin response to a serotonin agonist, $\mathrm{m}$-chlorophenylpiperazine (GarciaBorreguero et al, 1995). These responses are exaggerated during the winter depressive episode, returning to normal in summer or after phototherapy. These findings suggest a presynaptic serotonergic deficit during the episode, a hypothesis supported by studies showing a robust antidepressant effect of sertraline in SAD and by others showing that tryptophan depletion induces relapse in patients who have responded to phototherapy (Lam et al, 1996).

\section{Epidemiology}

Prevalence of around $4 \%$ was found in a telephone survey in Maryland, USA (Kasper et al, 1989b) and also in a recent postal survey in the UK (S. Barker, personal communication). This figure is higher than would be expected, given that many community surveys have found the prevalence of all depressive disorders to be 5-10\%. It may be that the methodology of these two studies has resulted in an inflated figure as both defined cases using the seasonality score on the Seasonal Pattern Assessment Questionnaire (SPAQ; Rosenthal et al, 1984) rather than DSM criteria. The SPAQ has also been used to demonstrate that in the USA prevalence of SAD rises with increasing latitude, which supports the hypothesis that environmental light conditions are important in its aetiology 
Table 1. Comparison of seasonal and nonseasonal depressive disorder

Seasonal Non-seasonal

Seasonal prominence

$$
\text { in winter }
$$

Mania in summer

Atypical symptoms

Response to light

$\begin{array}{ll}+++ & + \\ +++ & + \\ +++ & ++ \\ +++ & -\end{array}$

(Rosen et al, 1990). In contrast, a study in Iceland found the prevalence to be only $3.8 \%$, a lower figure than for any of the USA sites (Magnusson \& Stefannson, 1993). However, a further study of a well-circumscribed Icelandic community in Canada found an even lower prevalence of $1.2 \%$ (Magnusson \& Axelsson, 1993). Taken together, these studies on Icelanders support the association between latitude and prevalence of SAD, but also suggest the importance of cultural, ethnic and genetic factors.
The mean age at onset appears to be during the third decade, although there is a wide range. Indeed, there have been reports of SAD presenting in childhood, and many patients describe a seasonal pattern of mood change which began in their teenage years. Women are affected twice as often as men in both community and out-patient samples: the higher gender ratio reported in early studies appears to have been an artefact of recruitment methods which included advertising in women's magazines. A bipolar picture is seen in up to $30 \%$ of cases, with most of this subgroup satisfying criteria for bipolar II disorder (Winton \& Checkley, 1989).

\section{Clinical assessment}

Guidelines for the assessment of patients with SAD are shown in Fig. 1. It is also important to ask about the atypical depressive symptoms which occur in the majority of cases (Box 3). Although they are not required to make the diagnosis, they do predict

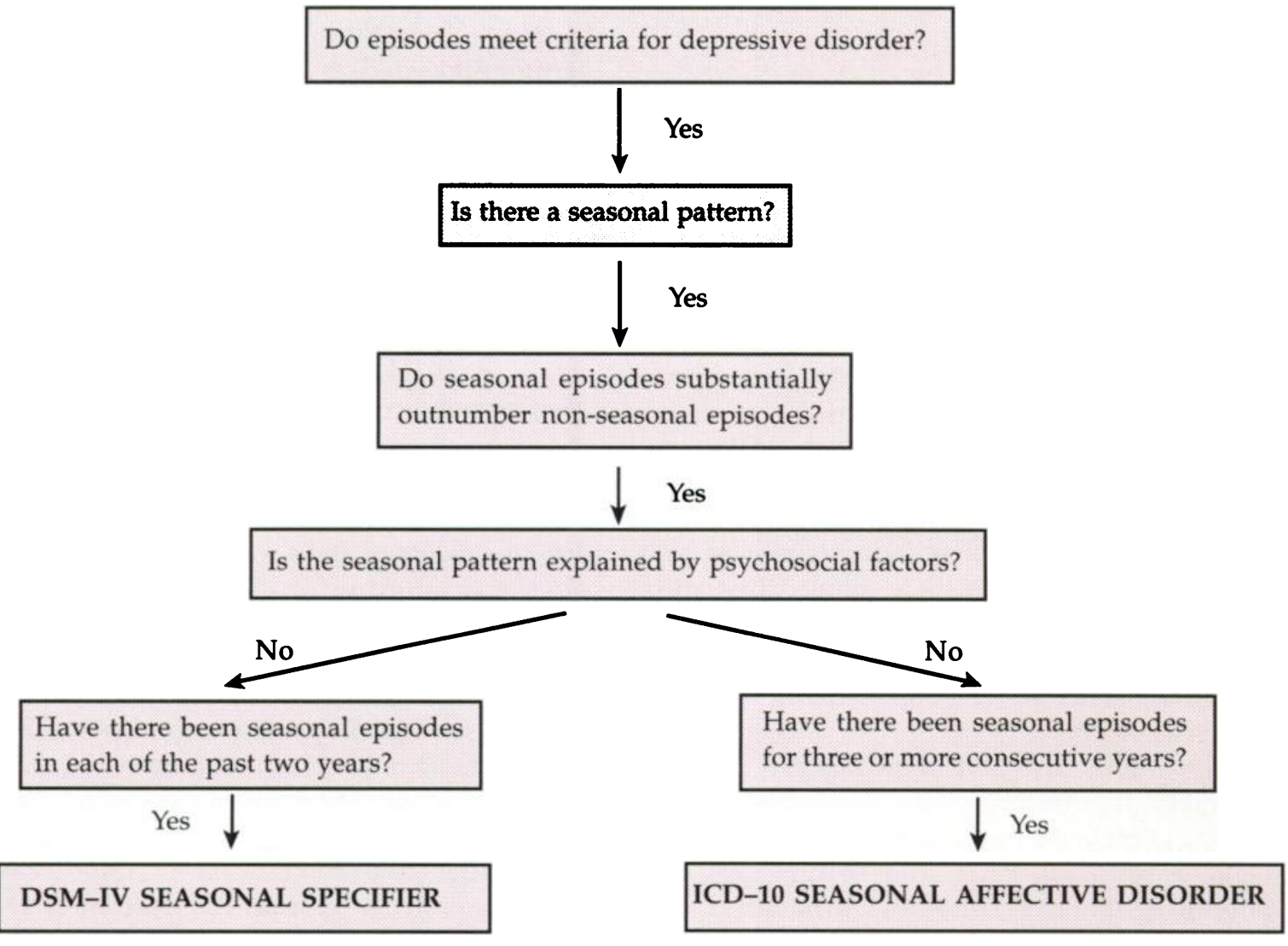


Box 3. Atypical symptoms

Increased sleep ${ }^{*}$

Increased appetite

Carbohydrate craving*

Weight gain

Fatiguability

Social withdrawal

Afternoon slump in energy*

* Predict good response to phototherapy

a good response to phototherapy (Terman et al, 1996).

Assessment of polarity is also important. Bipolar patients are those in whom there is conversion from depression to hypomania in spring. Spring hypomania in bipolar SAD is usually mild. However, some cases may require conventional antimanic treatment and there is also a small risk of treatment with phototherapy or antidepressants precipitating hypomanic episodes.

Clinical assessment may be augmented by use of the SPAQ (Rosenthal et al, 1984). This is a self-rated questionnaire which assesses the degree of seasonal variation of mood symptoms and determines at which time of year symptoms are at their worst. It is best given to patients before their first assessment as the information obtained usually saves time during the interview. The section of the SPAQ shown in Fig. 2 may also be used to derive a seasonality score, which provides a useful means of screening highrisk groups. Responses are scored from 0 to 4 for each item, generating a maximum total score of 24 . A score of 12 or more is predictive of SAD and scores of 8-11 are consistent with a diagnosis of subsyndromal SAD.

Standardised rating of symptom severity is best performed using the Structured Interview Guide for the Hamilton Depression Rating Scale, Seasonal Affective Disorder version (SIGH-SAD) in which seven items for assessment of atypical symptoms have been added to the standard 21-item scale (Williams et al, 1988). The SIGH-SAD should not be used as a diagnostic instrument but provides a useful measure of response to treatment.

It is extremely important to carry out a full diagnostic assessment in a patient presenting with possible SAD as many patients have vulnerability factors other than seasonality. As a result, treatment plans often include psychosocial measures in addition to the specific physical treatments available.

\section{Treatment}

\section{Phototherapy}

\section{Practical issues}

Phototherapy was developed specifically as a treatment for SAD. It is administered using specially designed light boxes which emit high intensity, visible-spectrum light. Treatment is given daily and, as with antidepressants, it may take up to two weeks before a therapeutic response is achieved, although many patients start to improve after 3-4 days. Maintenance treatment is then required daily for the rest of the winter, with treatment being stopped around the time spontaneous remission or conversion to hypomania usually occurs.

Light boxes cost $£ 75-200$ and are available from several suppliers in the UK. As it is impossible to predict with complete certainty whether a patient will respond to phototherapy, it is important that they have the opportunity of a trial of treatment before purchasing a light box. Ideally, light boxes should be available for patients to borrow from clinics for a trial period. Alternatively, several of the light box suppliers offer a free trial period of 2-3 weeks. Finally, there are hire schemes operating

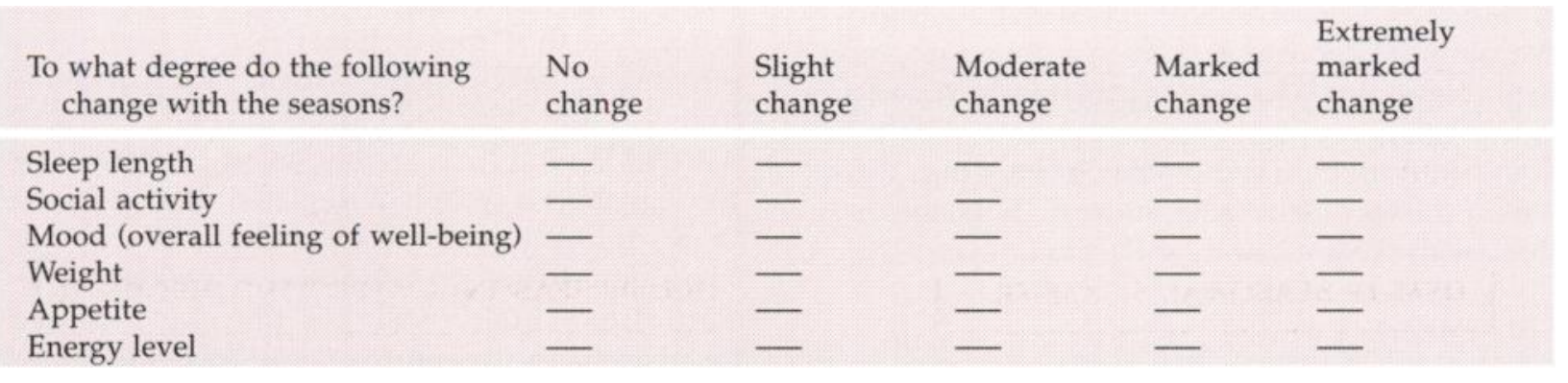

Iig. 2 Extract from the Seasonal Pattern Assessment Questionnaire 
in some regions, details of which can be obtained from the SAD Association (PO Box 989, Steyning, Sussex BN44 3HG). Factors to be considered when purchasing a light box include how portable it is, size and intensity of the light source, and cost.

\section{Administration of phototherapy}

Two intensities of light box are marketed, 2500 lux and 10000 lux. With 2500 lux boxes, patients usually require at least two hours of treatment daily to achieve, and then maintain, a therapeutic response. With 10000 lux, the required dose of phototherapy may be reduced to as little as $\mathbf{4 5}$ minutes daily. The main disadvantage of 10000 lux boxes is that they cost up to twice as much as 2500 lux boxes.

Early studies suggested that extension of the photoperiod (i.e. administering phototherapy at the start and at the end of the day) was necessary for a therapeutic response. This has not been supported by subsequent work which has shown that even phototherapy administered during the middle of the day can be effective. However, the optimum timing of phototherapy remains controversial. Some studies have found early morning treatment to be superior to evening treatment, whereas others have found no difference (Terman et al, 1989; Tam et al, 1995). On the balance of this evidence, we advise early morning treatment where possible, especially if other treatment conditions have failed to produce an adequate response. However, many patients find a combination of morning and evening phototherapy, or evening phototherapy alone, to be more convenient and just as effective. The only absolute rule is that phototherapy should never be given after $10.00 \mathrm{pm}$ as it has an alerting effect and will induce initial insomnia.

\section{Predictors of response}

The diagnosis of SAD itself predicts response to phototherapy, when compared with non-seasonal depressive disorders. Patients with atypical symptoms respond better to phototherapy and the symptoms with particular predictive value are marked in Box 3 (Terman et al, 1996). In contrast, a melancholic picture with classical biological symptoms of depression predicts a poorer response to phototherapy. What seems particularly important is the balance of symptoms: patients with predominantly atypical symptoms do better than those with a mixed picture who, in turn, do better than those with predominantly melancholic symptoms.

\section{Side-effects}

Phototherapy is usually well tolerated. Studies of side-effects have found eye strain, headache, insomnia and a subjective sense of tension to be reported by $10-20 \%$ of subjects (Levitt $e$ al, 1993). These symptoms are usually mild but if they are intolerable, there are several options which allow treatment to continue. The patient can sit slightly further away from the light box, thereby reducing the intensity of light they are exposed to. Alternatively, they can reduce the daily duration of phototherapy or they can split the daily dose between two or three sittings. In the case of insomnia, treatment should be moved to earlier in the day. Obviously, these strategies for dealing with side-effects may alter the therapeutic response but fortunately, side-effects usually improve with small changes to the treatment regime which have little effect on outcome. Even if substantial alterations are required, experimentation with different treatment regimes usually provides a satisfactory alternative.

Precipitation of hypomanic episodes by phototherapy has been reported in patients with a bipolar pattern. These episodes are usually mild and may be stopped by reducing the daily dose of phototherapy.

\section{Therapeutic mechanism}

The therapeutic mechanism of phototherapy is unknown and there has been much debate about whether it is merely an elaborate placebo. This hypothesis has been hard to refute because of the difficulty in designing satisfactory placebo conditions in phototherapy trials. However, a large number of open trials and trials comparing phototherapy against a variety of comparators have consistently shown it to have efficacy in the treatment of SAD (Terman et al, 1989; Tam et al, 1995). These findings, taken with the probable superiority of morning over evening light, suggest more than a placebo mechanism.

Phototherapy certainly has a biological effect, altering the amplitude and timing of melatonin secretion (Blear \& Rosenthal, 1989). However, these effects are not specific to SAD, are not correlated with treatment response and so do not explain the therapeutic mechanism. A more plausible hypothesis is that phototherapy acts through a serotonergic mechanism. Evidence for this comes from a recent study by Lam et al (1996). Subjects with SAD who had responded to phototherapy were given a tryptophan-depleted diet and were then randomised to receive either placebo or supplements of tryptophan, a precursor of serotonin. Those receiving placebo relapsed whereas the therapeutic response was maintained in those receiving tryptophan. A serotonergic mechanism is also supported by the finding that 
the exaggerated cortisol and prolactin responses to $\mathrm{m}$-chlorophenylpiperazine are reduced by phototherapy (Garcia-Borreguero, 1995).

\section{Other forms of phototherapy}

Because retinal stimulation by light is necessary for a therapeutic response to phototherapy, patients are required to face in the direction of the light box. In an attempt to overcome the inconvenience associated with this, light visors have been developed. These are attached to the patient's head and project light into their eyes, thereby allowing them to remain mobile during treatment. However, efficacy of light visors has not been demonstrated convincingly and the finding that treatment response is independent of light intensity suggested that any benefits of light visors were likely to be the result of a placebo effect (Rosenthal et al, 1993).

Dawn simulators are intended to reproduce the gradual increase of light intensity which occurs in the morning during summer (Avery et al, 1992). There is no convincing evidence for their efficacy in SAD although some patients find them a pleasant alternative to alarm clocks.

An alternative to daily phototherapy throughout each winter has been proposed by Partonen \& Lonnqvist (1996). They found that exposure to very high intensity light at the start of the winter prevented recurrence of SAD that winter. This study requires replication but offers an interesting alternative to the inconvenience of conventional phototherapy. Anecdotal support for its findings come from patient reports that a holiday to a sunny destination during the winter often produces symptom relief for weeks or months after their return.

\section{Medication}

There have been few trials of pharmacotherapy in SAD and sertraline is the only antidepressant for which efficacy has been clearly demonstrated: in a large, international multi-centre study, it was found to be superior to placebo across a range of outcome measures (Blashko et al, 1997). There are no theoretical reasons to believe that other selective serotonin reuptake inhibitors (SSRIs) should be less efficacious, but in the only other placebo-controlled study of conventional antidepressants in SAD, fluoxetine was not found to be superior to placebo (Lam et al, 1995). There have been no studies of tricyclic antidepressants in SAD: our clinical experience is that they may be useful in patients who have responded to them previously or in those who fail to respond to phototherapy or SSRIs.
In the only placebo-controlled trial of monoamine oxidase inhibitors in SAD, moclobemide was not found to be superior to placebo, but this trial had numerous methodological flaws (Lingjaerde et al, 1993). Some small studies have suggested that tryptophan may be of benefit, either alone or as an adjunct to phototherapy (McGrath et al, 1990). As discussed above, abnormalities of melatonin secretion in SAD have been demonstrated by some studies, prompting speculation that melatonin may be an effective treatment, but in practice it is devoid of any benefit.

As with phototherapy, antidepressant medication should be stopped around the time remission or conversion to hypomania usually occurs. Phototherapy may be safely combined with medication although there is a theoretical risk of over-arousal with activating antidepressants and, in patients with a bipolar pattern, of hypomania. In our experience, combined treatment may be useful in patients who have not responded to phototherapy or medication alone.

\section{Case history}

Mrs X. is a 35-year-old married woman. She presented in January with a three-month history of low mood, marked lethargy, hypersomnia, increased appetite and weight gain. She had experienced identical symptoms between October and April during eight of the past 10 winters. She had a depressive episode three summers ago following an enforced change of role at work. Apart from this, she had not been depressed outside the winter months. A diagnosis of SAD was made and she was lent a 10000 lux light box for a two week trial of early morning light. She responded well to this and made arrangements to purchase her own light box. She was advised to start reducing the daily duration of phototherapy in late March to avoid exacerbating the brief period of hypomania she usually experienced in spring.

She presented again three years later. She had been using her light box with good effect since she was last seen, starting treatment in late October when she noticed the first signs of relapse and continuing until the following March. However, she had increased her hours at work and no longer had time to use her light box in the morning. She was advised to try evening phototherapy but, as is sometimes the case, she found this much less effective than morning treatment. Therefore, she was started on sertraline to which she responded well.

\section{Prognosis}

About two-thirds of patients diagnosed with SAD will relapse the following winter. Longer-term 
follow-up studies have obtained broadly similar results, with $26-38 \%$ continuing to meet diagnostic criteria for $\mathrm{SAD}$, around $30 \%$ having recurrent episodes of non-seasonal depression and around $20 \%$ remaining well. In the largest of these studies, Thompson et al (1995) re-interviewed 93 subjects 5-8 years after they had been diagnosed as having SAD. They found that short duration of index episode and high frequency of seasonal episodes of depression predicted a continuing seasonal course. Another interesting finding in this study was that $8 \%$ of the subjects had emigrated at follow-up.

\section{Summer SAD}

There have been a few reports of patients with recurrent depressive episodes occurring during summer only. They have not shown the atypical symptom pattern seen in winter SAD and appear to respond best to conventional treatments for depressive disorder. Small studies have suggested taking cold showers, wearing sunglasses and avoiding sunlight and heat to be of benefit, but these all used highly flawed methodologies. At present, there is no convincing evidence to suggest that summer SAD is a distinct syndrome and so patients with this pattern should be treated in the same way as those with non-seasonal depressive disorder.

\section{Summary}

SAD is a relatively common subtype of mood disorder for which diagnostic criteria are included in DSM-IV and the research version of ICD-10. The syndrome has validity and serotonergic mechanisms appear to be important in its aetiology. Recognition of the condition is important as there is good evidence to support the effectiveness of phototherapy and sertraline in winter SAD.

\section{References}

Avery, D. H., Bolte, M.A. P., Cohen, S., et al (1992) Gradual versus rapid dawn simulation treatment of winter depression. Journal of Clinical Psychiatry, 53, 359-363.

Blashko, C. A. (1997) A double-blind placebo controlled study of sertraline in the treatment of out-patients with seasonal affective disorder. European Neuropsychopharmacology, 5, 258.

Blear, M. C. \& Rosenthal, N. E. (1989) Seasonal affective disorder and phototherapy. Report of a National Institute of Mental
Health sponsored workshop. Archives of General Psychiatry, 46, 469-474.

Dittman, V., Elster, K., Graw, P., et al (1994) Seasonal affective disorder: are the DSM-III-R criteria valid? Psychopathology, 27, 291-297.

Garcia-Borreguero, D., Jacobsen, F. M., Murphy, D. L., et al (1995) Hormonal responses to the administration of $\mathrm{m}$-chlorophenylpiperazine in patients with seasonal affective disorder and controls. Biological Psychiatry, 37, 740-749.

Kasper, S., Rogers, B., Yancey, A., et al (1989a) Phototherapy in individuals with and without subsyndromal seasonal affective disorder. Archives of General Psychiatry, 46, 837-844.

-, Wehr, T., Bartako, J. J., et al (1989b) Epidemiological findings of seasonal changes in mood and behaviour: a telephone survey of Montgomery County, Maryland. Archives of General Psychiatry, 46, 823-833.

Lam, R. W., Gormon, C. P., Michalon, M., et al (1995) Multicenter placebo-controlled study of fluoxetine in seasonal affective disorder. American Journal of Psychiatry, 152, 1765-1770.

- , Athanasios, P. Z., Grewal, A., et al (1996) Effects of rapid tryptophan depletion in patients with seasonal affective disorder in remission after light therapy. Archives of General Psychiatry, 53, 41-44.

Levitt, A. J., Joffe, R. T., Moul, D. E., et al (1993) Side effects of light therapy in seasonal affective disorder. American Journal of Psychiatry, 150, 650-652.

Lewy, A. J., Sack, R. L., Singer, C. M., et al (1987) The phase shift hypothesis for bright light's therapeutic mechanism of action: theoretical considerations and experimental evidence. Psychopharmacology Bulletin, 23, 349-353.

Lingjaerde, O., Reichborn-Kjennerud, T. \& Haggag, A. (1993) Treatment of winter depression in Norway. II: A comparison of the selective monoamine oxidase A inhibitor moclobemide and placebo. Acta Psychiatrica Scandinavica, 88, 372-380.

Magnusson, A. \& Stefannson, J. G. (1993) Prevalence of seasonal affective disorder in Iceland. Archives of General Psychiatry, 50, 941-946.

- \& Axelsson, J. (1993) The prevalence of seasonal affective disorder is low among descendants of Icelandic immigrants in Canada. Archives of General Psychiatry, 50, 947-951.

McGrath, R. E., Buckwald, B. \& Resnick, E. V. (1990) The effect of l-tryptophan on seasonal affective disorder. Journal of Clinical Psychiatry, 51, 162-163.

Partonen T. \& Lonnqvist, J. (1996) Prevention of winter seasonal affective disorder by bright light treatment. Psychological Medicine, 26, 1075-1080.

Rosen, L. N., Targum, S. D., Terman, M., et al (1990) Prevalence of seasonal affective disorder at four latitudes. Psychiatry Research, 31, 131-144.

Rosenthal, N. E., Sack, D. A., Gillin, J. C., et al (1984) Seasonal affective disorder: a description of the syndrome and preliminary findings with light therapy. Archives of General Psychiatry, 41, 72-80.

-, Moul, D. E. \& Hellekson, C. J. (1993) A multicenter study of the light visor for seasonal affective disorder: no difference in efficacy found between two different intensities. Neuropsychopharmacology, 8, 151-160.

Tam, E. W., Lam, R. W. \& Levitt, A. J. (1995) Treatment of seasonal affective disorder: a review. Canadian Journal of Psychiatry, 40, 457-466.

Terman, M., Amira, L., Terman, J. S., et al (1996) Predictors of response and non-response to light treatment for winter depression. American Journal of Psychiatry, 153, 1423-1429.

-, Terman, J. S., Quitkin, F. M., et al (1989) Light therapy for seasonal affective disorder. Neuropsychopharmacology, 2, 1-22.

Thompson, C. (1989) Seasonality of depression. In Seasonal Affective Disorder (eds C. Thompson \& T. Silverstone), pp. 1-17. London: CNS Publishers.

—, Stinson, D. \& Smith, A. (1990) Seasonal affective disorder and season dependent abnormalities of melatonin secretion by light. Lancet, 336, 703-706.

-, Raheja, S. K. \& King, E. A. (1995) A follow-up study of seasonal affective disorder. British Journal of Psychiatry, 167, 380-384.

Williams, J. B. W., Link, M. J., Rosenthal, N.E., et al (1988) Structured interview guide for the Hamilton Depression 
Rating Scale, Seasonal Affective Disorders Version (SIGHSAD). New York: New York Psychiatric Institute.

Winton, F. \& Checkley S. A. (1989) Clinical characteristics of patients with seasonal affective disorder. In Seasonal Affective Disorder (eds C. Thompson \& T. Silverstone), pp. 59-67. London: CNS Publishers.

\section{Multiple choice questions}

1. Seasonal affective disorder:
a is a recurrent depressive disorder
b usually shows a bipolar pattern
c has an equal incidence in both genders
$\mathrm{d}$ has a prevalence of $0.5 \%$.

2. SAD should not be diagnosed if:

a atypical symptoms are absent

b only three seasonal episodes have occurred

c there is a history of non-seasonal depressive episodes

d depressive episodes are secondary to seasonal unemployment.

3. The following are first line treatments for SAD:
a phototherapy
b tricyclic antidepressants
c SSRIs
d melatonin.

4. Regarding phototherapy:
a sunglasses should be worn to protect the eyes during treatment
b endogenous symptoms predict a good response
c headaches are a recognised side-effect
d treatment is given once weekly.

5. A continuing seasonal pattern of depression is predicted by:
a age over 40 years
b an index episode lasting five months or more
c seasonal episodes most years
d migration towards the equator.

\begin{tabular}{|c|c|c|c|c|}
\hline \multicolumn{5}{|c|}{ MCQ answers } \\
\hline 1 & 2 & 3 & 4 & 5 \\
\hline a $T$ & a $F$ & a $T$ & a F & a $F$ \\
\hline b $F$ & b F & b F & b F & b $F$ \\
\hline c $F$ & c $F$ & c $\mathrm{T}$ & c $\mathrm{T}$ & c $\mathrm{T}$ \\
\hline d F & d $T$ & d F & d F & d F \\
\hline
\end{tabular}

